
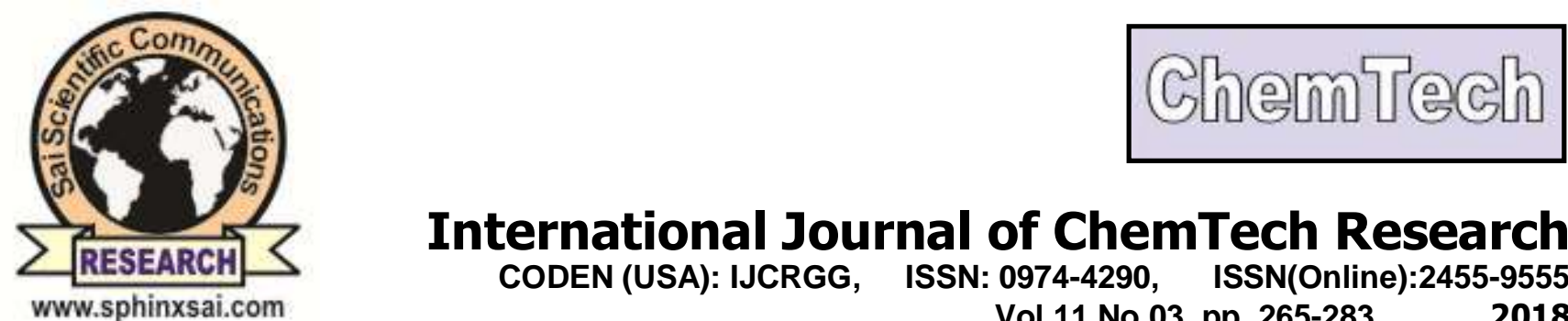

International Journal of ChemTech Research CODEN (USA): IJCRGG, ISSN: 0974-4290, ISSN(Online):2455-9555

Vol.11 No.03, pp 265-283,

2018

\title{
Biocompatible Silver Nanoparticles synthesized from Andrographis echioides and its Antioxidant and Antidiabetic effects on STZ induced Diabetic Rats
}

\author{
Nirubama Kumar* \\ GRD Bio Clinical Research, R\&D Manager, Rasipuram, Namakkal, India
}

\begin{abstract}
Diabetes mellitus is a chronic metabolic disorder characterized by complete or relative lack of insulin, or insulin resistance. Metal nanoparticles preparation using plant is an important branch of biosynthesis processes. Andrographis echioides (L.) Nees. (Family: Acanthaceae), also known as Indoneesiella echioides (L.) which is commonly known as false water willow. Various biochemical Parameters, Enzymic and non-enzymic antioxidants were analyzed in the liver, kidney, pancreas as per the standard procedures. Administration of AgNPs - hydroalcholoic extract of A. echioides (AgNPs- HEAE) significantly lowers the blood glucose level, gluconeogenic enzymes such as glucose-6-phosphatase and fructose-1, 6bisphosphatase whereas increases the activities of hexokinase, glucose-6 phosphatase dehydrogenase and GSH via by increasing level of insulin. AgNPs- HEAE reduces the total cholesterol and triglycerides, decreases the LDL-C, VLDL-C and it considerably increase the level of HDL-C. In plasma and tissues i.e. liver, kidney, pancreas, AgNPs- HEAE increased catalase activity and GSH level and decreased lipid peroxidation, and increases the enzymatic and non enzymatic antioxidants levels. Furthermore, the histological damages in pancreas, liver and kidney tissues were reduced. These results indicated a good hypoglycemic and antioxidant activity of AgNPs- HEAE. The overall results are suggestive that AgNPs hydroalcholoic extract of $A$. echioides act as efficient free radical scavenger intercept those radicals which are involved in drug metabolism by microsomal enzymes. To best of our knowledge this is the first report of green synthesis of AgNPs- HEAE antioxidant and antidiabetic properties.
\end{abstract}

Keywords : Oxidative stress; Silver Nanoparticles (AgNps); Biochemical estimations Andrographis echioides; Histopathology.

\section{Introduction}

Diabetes mellitus (DM) is an inherited, chronic disorder in the endocrine system that constitutes a main public health problem all over the worldwide [1]. DM is the heterogeneous metabolic disorder characterized by altered carbohydrate, lipid and protein metabolism. It is a primary source of morbidity and mortality in human populations that is defined as a group of metabolic diseases manifest by hyperglycemia which results from defects in insulin production and insulin action. In India the burden of DM is increase dramatically the year

International Journal of ChemTech Research, 2018,11(03): 265-283.

DOI : http://dx.doi.org/10.20902/IJCTR.2018.110331 
2010, it was estimated that more than 200 million people worldwide had DM and 300 million will subsequently have the disease by 2025 [2]. Hyperglycemia is a hallmark for the growth of many complications in diabetics like retinopathy, nephropathy, neuropathy and angiopathy. This plant based, traditional medicine system continues to play an essential role in healthcare, with about $80 \%$ of the world's inhabitants relying mainly on traditional medicines for their primary healthcare [3].

Insulin is an chief signaling molecule required by almost all of the cells, but its major targets are liver, fat and muscle cells. For these cells, insulin has the following function: stimulates liver and muscle cells to store glucose in glycogen, stimulates fat cells to form fats from fatty acids and glycerol, activate liver and muscle cells to make proteins from amino acids, and inhibits the liver and kidney cells from making glucose from intermediate compounds of metabolic pathways (gluconeogenesis) [4].

The nanotechnology is commonly defined as the manipulation of mater about the nanometers order (1$100 \mathrm{~nm}$ ) to produce materials with novel properties and functions with a potential wide range to applications. The nanomedicine is the application of the nanotechnology to medicine. Because of the similarity in the domain of size of nanotechnology with the biological structures and definite functional properties it is likely to make important advances in the areas of gene therapy, imaging and novel drug discovery and drug delivery in the treatment of diseases like diabetes and cancer.

The development of metal NPs stabilized and synthesized using green or chemical reduction methods have involved significance in the clinical field for its potential effects or toxicological that can have in the biological systems. In experimental diabetes, it has reported that $\mathrm{ZnO}$ and silver NPs decrease the glucose levels and increase the production of insulin. The bio-compatible NPs synthesized from plant extracts can contribute to toxic compounds elimination. Today, the diabetes investigations are focused in improve the clinical diagnosis, in the development of blood glucose biosensors and in the control and treatment with recent progress in the oral therapy trying to improve the bioavailability bioactivity and drug delivery. Recently, it has been reported that insulin-chitosan nano structured complexes improve the efficiency of oral insulin delivery. Another interesting study reported that carbon nanotubes functionalized with nicotinamide increase the production of insulin in pancreatic beta cell generating a new expectative in the antidiabetic therapy.

Streptozotocin (STZ) is a permanent diabetes inducing drug. It is synthesized by a strain of the soil microbe Streptomyces achromogenes (gram positive bacterium) with broad spectrum of antibacterial properties [5]. STZ is an unusual amino glycoside containing a nitrosoamino group discovered in 1959 as an antibiotic, now marketed as a generic drug. It has been widely used for inducing diabetes in the experimental rats through its toxic effects on pancreatic cells and it is well studied that exposure of the insulin secreting cells to STZ results in DNA damage in pancreatic cells through the formation of alkylating free radicals leading to hasty necrosis of the cells, thereby the rate of insulin synthesis and secretion is diminished [6]. Glibenclamide is often used as a reference drug in STZ-induced moderate diabetic model. Though sulphonylureas are valuable in treatment of diabetes, their use is restricted by their limited action and side effects (hypoglycaemia and liver problems) [7].

Reduced hemoglobin concentrations are common findings in diabetic patients [8]. The liver plays a major role in the regulation of carbohydrate metabolism, as it uses glucose as a fuel, it has the capability to store glucose as glycogen and also synthesize glucose from non-carbohydrate sources. This key function of liver makes it vulnerable to diseases in subjects with metabolic disorders, particularly diabetes. [9] Increased activities of liver enzymes such as aspartate aminotransferase (AST) and alanine aminotransferase (ALT) are indicators of epatocellular injury. Increased activity of these markers is associated with insulin resistance [10], metabolic syndrome and type 2 diabetes [11]. The typical lipid disorder in patient with diabetes, diabetic dyslipidemia, is characterized by elevated triglycerides, low levels of HDL cholesterol and increased numbers of small, dense LDL particles [12]. Hyperuricemia-uric acid is end product of purine metabolism, it is filtered in glamorous filtration and excretion in urine [13].

In humans everyday activities oxidation plays important role as there is antioxidant defense mechanism present such as lycopene, beta carotene, glutathione, flavonoid, selenium natural vitamin such as vitamin E, vitamin A antioxidant enzymes such as catalase peroxidase, glutathione-S-transferase finish the accessibility of peroxidase as hemeprotein, iron, copper ions. In recent studies there is strong relation found between oxidative stress and reactive oxygen species for human disorders/diseases. So antioxidants and free radicals studies are very important in today's research for understanding the relationships of diseases such as cancer, neurodegenerative diseases, diabetes mellitus and cardiac arrest [14]. 
Free radicals are defined as atoms or molecules that contain one or more unpaired electrons, making them unstable and highly reactive. The most important ROS are the superoxide anion radicals, hydrogen peroxide $\left(\mathrm{H}_{2} \mathrm{O}_{2}\right)$, alkoxyl $(\mathrm{RO})$, peroxyl (ROO) and hydroxyl radicals $(\mathrm{OH})$ [15] and hypochlorous acid $(\mathrm{HOCl})$, other non-oxygen species existing as reactive nitrogen species (RNS) such as nitric oxide (NO) and peroxy nitrite (ONOO) have also important bioactivity [16]. Oxidative stress plays a pivotal role in cellular injury from hyperglycemia. High glucose level can stimulate free radical production. Weak defense system of the body becomes unable to counteract the enhanced ROS generation and as a result condition of imbalance between ROS and their protection occurs which leads to domination of the condition of oxidative stress [17].

Antioxidant has been defined as any substance that delays or inhibits oxidative damage to a target molecule [18]. In generally all the antioxidants influence the redox status, thereby protecting cells against ROS under certain circumstances, while promoting ROS generation in others. Antioxidants are of two types namely enzymatic antioxidant such as SOD [19] CAT, GPx [20] and non-enzymatic antioxidants such as GSH, ascorbic acid (Vitamin C), $\alpha$-tocopherol (Vitamin E) [21], $\beta$-carotene (Vitamin A), estrogen [22] and flavonoids [23]. Andrographis echioides is a species of Andrographis commonly known as belonging to the family Acanthaceae, is a dense herb found in plains and waste lands which fruits throughout the year. Andrographis echioides has been reported anti-inflammatory, antimicrobial, anthelmintic, antioxidant and larvicidal activities [24].

The literature survey revealed that there are no scientific studies carried out regarding free radical and antioxidant properties of Andrographis echiodies. Hence, the present is focused to evaluate the antioxidant, hepatoprotective and antidiabetic analysis of hydroalcholic extract of AgNps- Andrographis echiodies.

Based on these considerations, the present study was designed to investigate the effects of a hydroalcholic extract of AgNps- Andrographis echiodies on the antidiabetic and oxidative stress induced by STZ induced diabetes in an animal model using male Wistar rats. Histopathological studies were also carried out to assess the effect of hydroalcholic extract of AgNps- Andrographis echiodies on liver, kidney and pancreatic cells against STZ damage in rats.

\section{Materials and Methods}

\subsection{Experimental animals}

Male albino rats of the Wistar strain, weighing 150-200 gm were obtained from Sri Venkateswara Enterprises, Bangalore. The rats were housed in a well-ventilated experimental animal house under constant environmental and adequate nutritional conditions throughout the period of the experiment. Feeding of rats was done ad libitum, along with drinking water. All the experiments were carried out according to the guidelines of the Committee for the Purpose of Control and Supervision of Experiments on Animals (CPCSEA), New Delhi, India and approved by the Institutional Animal Ethical Committee of Muthayammal college of Arts and Science (Reg. No. 1416/a/11/CPCSEA).

\subsection{Induction of diabetes mellitus}

Non-insulin dependent diabetes was induced in overnight fasted male albino Wistar rats by a single intraperitonial (i.p.) injection of $40 \mathrm{mg} / \mathrm{kg}$ STZ. STZ freshly dissolved in $0.1 \mathrm{M}$ citrate buffer (pH 4.5) [25]. After 72 hours, diabetic rats were identified by measuring fasting plasma glucose (FPG) levels. The rats with blood glucose concentration more than $200 \mathrm{mg} / \mathrm{dL}$ were used in this study.

\subsection{Preparation of Plant Extract}

Exactly $200 \mathrm{~g}$ of shade dried coarsely powdered whole plant of A. echioides was extracted with $500 \mathrm{ml}$ of $80 \%$ aqueous ethanol by maceration at room temperature for 72 hours. After extraction, the extract was filtered, concentrated to dryness in rotavapour under reduced pressure and controlled temperature $\left(40-500^{\circ} \mathrm{c}\right)$. Dark yellowish brown colour residue was obtained and it was coded as A. echioides. The residue was then stored in desiccators.

\subsection{Synthesis of silver nanoparticles}

$3 \mathrm{mM}$ solution of silver nitrate was prepared. $20 \mathrm{ml}$ of the plant extract was mixed with $80 \mathrm{ml}$ of $3 \mathrm{mM}$ of 
silver nitrate solution. The colour changed from yellow to reddish brown colour indicating the formation of silver nanoparticles. The AgNps thus obtained was purified by repeated centrifugation at $7000 \mathrm{rpm}$ for 10 minutes. The precipitate was collected directly. Andrographis echioides (AgNps - HEAE) was dissolved in $0.2 \%$ dimethyl sulfoxide (DMSO) and administrated to rats orally using an intragastric tube [26].

\subsection{Experimental design}

In this study, a total of 30 rats were divided into six groups of six rats in each group and had free access to food and water and were treated for 21 days as follows: Group I - Control rats, Group II - rats were induced for STZ, Group III - orally administration of STZ along with AgNps - hydroalcoholic extract of A. echioides stz $(200 \mu \mathrm{g} / \mathrm{kg} / \mathrm{bw})$, Group IV - orally administration of STZ along with AgNps - hydroalcoholic extract of $A$. echioides $(400 \mu \mathrm{g} / \mathrm{kg} / \mathrm{bw})$ and Group $\mathrm{V}$ - orally administration of standard drug glibencilamide. At the end of the experimental period, all the rats were fasted overnight, anaesthetized using pentobarbital sodium $(35 \mathrm{mg} / \mathrm{kg}$ b.w., intramuscular injection), and sacrificed by cervical decapitation. The blood samples were collected in the test tubes containing potassium oxalate and sodium fluoride (3:1) as anticoagulant for the separation of plasma, serum, erythrocytes. The liver, kidney and pancreas were immediately dissected out, washed in ice-cold saline to remove the blood, and pat dried and weighed.

The liver, kidney and pancreas were weighed and $10 \%$ of tissue homogenate was used for the analysis of lipid peroxidation, enzymatic and non-enzymatic antioxidants. Histopathological studies were carried out by the technique of [25]. The liver, kidney and pancreas tissue was preserved in $10 \%$ commercial formalin immediately on removal from the animal.

\subsection{Biochemical estimations}

Glucose was estimated by the method of Trinder using reagent Kit (1969), [27] Hemoglobin in the blood was estimated by the method of Drabkin and Austin (1932) [28] Glycosylated hemoglobin in the blood was estimated by the method of Sudhakar Nayak and Pattabiraman, (1981).[29] Protein in the tissues was determined after trichloro acetic acid precipitation by the method of Lowry et al., (1951)[30]. Liver glycogen was extracted and estimated by the method of Morales et al., (1975).[31] Hexokinase D was assayed by the method of Brandstrup et al., (1957).[32] Glucose 6-phosphatase was assayed by the method of Koide and Oda (1959).[33] Fructose 1, 6-bisphosphatase was assayed by the method of Gancedo and Gancedo (1971).[34] Glucose 6-phosphate dehydrogenase in the erythrocytes and liver was assayed by the method of Bergmeyer (1984).[35] Lipids were extracted from plasma and tissues by the method of Folch et al., (1951)[36] Total cholesterol in the plasma, erythrocytes and tissues was estimated by the enzymic method described by Allain $e t$ al., (1974).[37] HDL-cholesterol was estimated using the diagnostic kit based on the enzymic method described by Izzo et al., (1981)[38]. Estimation of VLDL and LDL-cholesterol (Friedewald et al., 1972) [39] Free fatty acids in the plasma and tissues were estimated by the method of Falholt et al., (1973).[40] Triacylglycerol in the plasma and tissues were estimated using the diagnostic kit based on the enzymic method described by McGowan et al.,(1983).[41] Phospholipids in the plasma, erythrocytes and tissues were estimated by the method of (Zilversmit and Davis 1950).[42] Total protein and albumin in the serum were estimated by Biuret method (Reinhold, 1953).[43] Serum aspartate aminotransferase was assayed by using the diagnostic kit based on the method of Reitman and Frankel (1957). [44] Serum alanine aminotransferase was assayed by using the diagnostic kit based on the method of Reitman and Frankel (1957). Plasma alkaline phosphatase was estimated by using the diagnostic kit based on Kind and King's method (1954). [45] The enzyme activity was assayed according to the method of Rosalki and Rau (1972). [46] Urea in the plasma and urine was estimated by using the diagnostic kit based on the method of Fawcett and Scott (1960). [47]Uric acid in the plasma and urine was estimated by using the diagnostic kit based on the enzymic method described by Caraway (1955).[48] Creatinine in the plasma and urine was estimated using the diagnostic kit based on the method of Tietz (1987) [49] using Jaffe's (1886) color reaction. Plasma thiobarbituric acid reactive substances (TBARS) were estimated by the method of Yagi et al. [50]. The concentration of TBARS in the tissue was estimated by the method of Fraga et al. [51]. The levels of lipid hydroperoxides (HP) were estimated by the method of Jiang et al. [52]. The activity of SOD was assayed according to the procedure of Kakkar et al. [53]. The activity of catalase was assayed by the method of Sinha et al. [54]. The activity of GPx was assayed by the method of Rotruck et al. [55]. The level of GSH was estimated by the method of Ellman et al. [56]. The levels of vitamin C were estimated by the method of Omaye et al. [57] The levels of vitamin E were estimated by the method of Baker et al. [58]. 


\subsection{Histopathology}

Tissues (liver, kidney, and Pancreas) obtained from all experimental groups were washed immediately with saline and then fixed in $10 \%$ buffered neutral formalin solution. After fixation, the tissue was processed by embedding in paraffin. Then, the tissue was sectioned and stained with Haematoxylin and Eosin (H\&E) and examined under high power microscope and photomicrographs were taken.

\subsection{Statistical analysis}

Statistical analysis was performed by one way analysis of variance (ANOVA) followed by Duncan's Multiple Range Test (DMRT). Using Statistical Package for the Social Sciences (SPSS) software package version 16.00. $\mathrm{P}$ values $<0.05$ were considered significant.

\section{RESULTS}

\subsection{Effect of AgNps-HEAE on plasma glucose and insulin levels}

Fig. 1 illustration that the effect of AgNps-HEAE were determined in the levels of plasma glucose and insulin in control and experimental rats. The glucose level significantly increased and insulin level significantly decreased were observed in STZ induced diabetic rats. Diabetic rats treated with oral supplementation of AgNpsHEAE significantly upturned the glucose and insulin levels. There were no changes in glucose and insulin levels detected between control, standard drug glibencilamide and AgNps-HEAE alone treated rats.

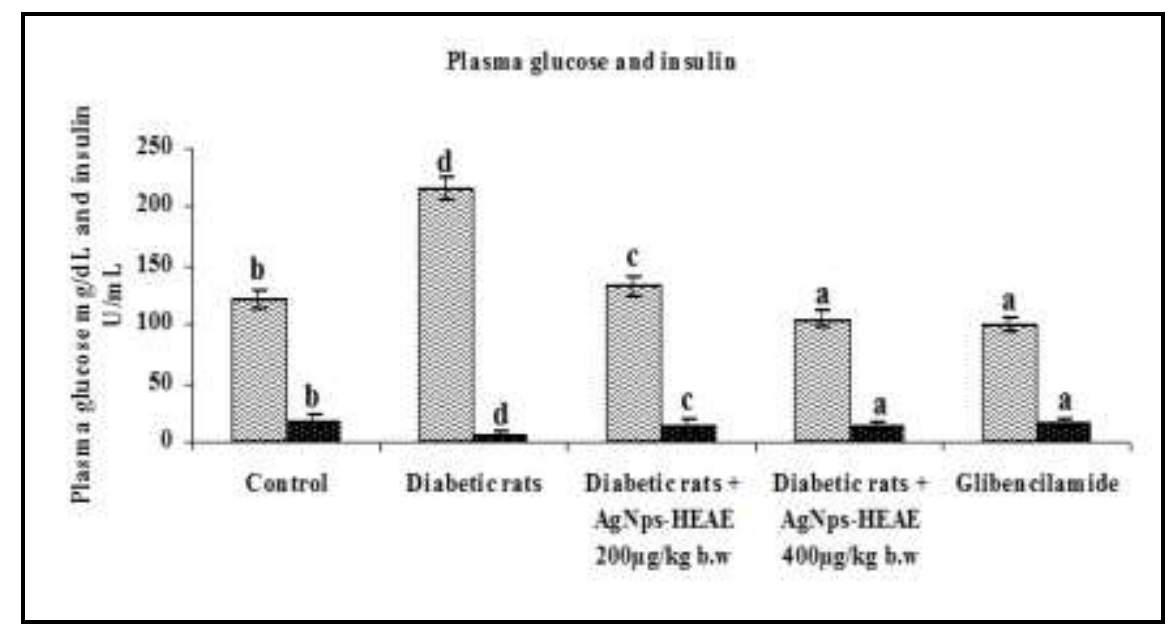

Fig 1: Plasma glucose and insulin levels

Table 1 Effect of AgNps-HEAE on levels of $\mathrm{HbA1c}, \mathrm{Hb}$, protein.

\begin{tabular}{|l|l|l|l|l|l|}
\hline Groups & Control & $\begin{array}{l}\text { Diabetic } \\
\text { rats }\end{array}$ & $\begin{array}{l}\text { Diabetic rats } \\
+\quad \text { AgNps- } \\
\text { HEAE } \\
\mathbf{2 0 0 \mu g / k g ~ b . w ~}\end{array}$ & $\begin{array}{l}\text { Diabetic rats } \\
+ \text { AgNps- } \\
\text { HEAE } \\
\mathbf{4 0 0 \mu g / k g ~ b . w ~}\end{array}$ & Glibencilamide \\
\hline Ure a (mg/dl) & $17.33 \pm 0.46$ & $75.23 \pm 0.89^{*}$ & $33.33 \pm 0.51^{\dagger}$ & $19.83 \pm 0.31^{\S}$ & $18.00 \pm 0.26^{\S}$ \\
\hline $\begin{array}{l}\text { Uric Acid } \\
(\mathrm{mg} / \mathrm{dl})\end{array}$ & $1.48 \pm 0.06$ & $3.39 \pm 0.05^{*}$ & $2.02 \pm 0.02^{\dagger}$ & $1.72 \pm 0.03^{\S}$ & $1.52 \pm 0.02^{\S}$ \\
\hline $\begin{array}{l}\text { Creatinine } \\
(\mathrm{mg} / \mathrm{dl})\end{array}$ & $0.68 \pm 0.02$ & $2.88 \pm 0.02^{*}$ & $1.50 \pm 0.01^{\dagger}$ & $1.04 \pm 0.01^{\S}$ & $0.80 \pm 0.02^{\S}$ \\
\hline $\begin{array}{l}\text { Hemoglobin } \\
(\mathrm{g} / \mathrm{dl})\end{array}$ & $15.32 \pm 0.08$ & $9.35 \pm 0.13^{*}$ & $11.73 \pm 0.05^{\dagger}$ & $13.35 \pm 0.06^{\S}$ & $14.46 \pm 0.07^{\S}$ \\
\hline HbA1C (\%) & $4.66 \pm 0.04$ & $8.40 \pm 0.05^{*}$ & $6.20 \pm 0.03^{\dagger}$ & $5.46 \pm 0.05^{\S}$ & $5.29 \pm 0.04^{\S}$ \\
\hline Protein (mg/dl) & $6.42 \pm 0.06$ & $3.34 \pm 0.08^{*}$ & $4.51 \pm 0.04^{\dagger}$ & $5.81 \pm 0.04^{\S}$ & $6.23 \pm 0.02^{\S}$ \\
\hline
\end{tabular}


Antidiabetic effect of AgNps-HEAE on urea, uric acid, creatinine, hemoglobin, HbA1c and protein in STZ induced Diabetic Rats. Values presented as Mean \pm standard deviation $(n=6)$. Values not sharing a common superscript ${ }^{\dagger} \mathrm{p}<0.05$ and ${ }^{\S} \mathrm{p}<0.01$ (DMRT).

\subsection{Effect of AgNps-HEAE on levels of HbA1c, Hb, protein.}

The effect of AgNps-HEAE in the levels of $\mathrm{HbAlc}, \mathrm{Hb}$ and protein in control and experimental rats. The HbA1c level significantly increased and significantly reduced $\mathrm{Hb}$ and protein were observed in STZ induced diabetic rats. Diabetic rats treated with oral administration of AgNps-HEAE significantly decreased the level of $\mathrm{HbA1c}$ and significantly increased the levels of $\mathrm{Hb}$ and protein. No changes in the levels of HbAlc, $\mathrm{Hb}$ and protein was detected in control, glibencilamide standard drug and AgNps-HEAE alone treated rats (Table. 1).

3.3. Effect of AgNps-HEAE on activities of hexokinase, glucose 6 phosphatase, fructose 1,6 bisphosphatase and glucose 6 phosphate dehydrogenase of liver and kidney.

Table. 2 shows the activities of hexokinase, glucose 6 phosphatase, fructose 1, 6 bisphosphatase and glucose 6 phosphate dehydrogenase in control and experimental rats of liver and kidney. The hexokinase, glucose 6 phosphatase, fructose 1, 6 bisphosphatase and glucose 6 phosphate dehydrogenase levels diminished were observed in STZ induced diabetic rats of liver and kidney. Diabetic rats treated with oral administration of AgNps-HEAE significantly improved the levels of hexokinase, glucose 6 phosphatase, fructose 1, 6 bisphosphatase and glucose 6 phosphate dehydrogenase. There is no changes in enzyme activity was observed with control, standard drug glibenclamide and AgNps-HEAE alone treated experimental rats of liver and kidney.

Table. 2 Effect of AgNps-HEAE on lipid profile in STZ induced levels of plasma

\begin{tabular}{|c|c|c|c|c|c|c|}
\hline Groups & Tissues & Control & Diabetic rats & $\begin{array}{l}\text { Diabetic rats } \\
+\quad \text { AgNps- } \\
\text { HEAE } \\
200 \mu \mathrm{g} / \mathrm{kg} \\
\text { b.w }\end{array}$ & $\begin{array}{l}\text { Diabetic rats } \\
+\quad \text { AgNps- } \\
\text { HEAE } \\
400 \mu \mathrm{g} / \mathrm{kg} \\
\end{array}$ & Glibencilamide \\
\hline \multirow{2}{*}{$\begin{array}{l}\text { Hexokinase } \\
\text { (Unit/h/mg } \\
\text { protein) }\end{array}$} & Liver & $129.25 \pm 1.01$ & $35.75 \pm 1.98^{*}$ & $90.20 \pm 1.10^{\dagger}$ & $117.70 \pm 3.26^{\S}$ & $125.95 \pm 1.79^{\S}$ \\
\hline & Kidney & $96.80 \pm 1.10$ & $12.65 \pm 1.01^{*}$ & $55.55 \pm 1.01^{\dagger}$ & $91.96 \pm 2.68^{\S}$ & $94.60 \pm 3.37^{\S}$ \\
\hline \multirow{2}{*}{$\begin{array}{l}\text { Glucose 6 } \\
\text { Phosphatase } \\
\text { (Unit/h/mg } \\
\text { protein) }\end{array}$} & Liver & $80.56 \pm 1.03$ & $27.43 \pm 0.84^{*}$ & $63.54 \pm 0.71^{\dagger}$ & $73.96 \pm 1.04^{\S}$ & $75.35 \pm 0.99^{\S}$ \\
\hline & Kidney & $62.85 \pm 0.84$ & $17.01 \pm 3.20^{*}$ & $30.90 \pm 1.25^{\dagger}$ & $43.75 \pm 1.20^{\S}$ & $57.29 \pm 1.92^{\S}$ \\
\hline \multirow{2}{*}{$\begin{array}{l}\text { Fructose 1,6 } \\
\text { Bisphosphatase } \\
\text { (Unit/h/mg } \\
\text { protein) }\end{array}$} & Liver & $158.33 \pm 4.81$ & $48.61 \pm 2.56^{*}$ & $98.61 \pm 4.52^{\dagger}$ & $133.33 \pm 2.15^{\S}$ & $147.22 \pm 5.96^{\S}$ \\
\hline & Kidney & $109.72 \pm 2.56$ & $23.61 \pm 2.56^{*}$ & $63.89 \pm 4.12^{\dagger}$ & $100.00 \pm 2.15^{\S}$ & $104.17 \pm 1.86^{\S}$ \\
\hline \multirow{2}{*}{$\begin{array}{l}\text { Glucose 6 } \\
\text { Phosphate } \\
\text { dehydrogenase } \\
\text { (Unit/h/mg } \\
\text { protein) }\end{array}$} & Liver & $142.90 \pm 1.90$ & $50.23 \pm 1.61^{*}$ & $87.86 \pm 1.93^{\dagger}$ & $124.24 \pm 2.01^{\S}$ & $128.13 \pm 1.85^{\S}$ \\
\hline & Kidney & $111.96 \pm 2.41$ & $31.72 \pm 0.90^{*}$ & $61.11 \pm 1.27^{\dagger}$ & $94.39 \pm 1.21^{\S}$ & $100.30 \pm 2.64^{\S}$ \\
\hline
\end{tabular}

Antidiabetic effect of AgNps-HEAE on hexokinase, glucose 6 phosphatase, fructose 1,6 bis phosphatase and glucose 6 phosphate dehydrogenase in STZ induced Diabetic Rats. Values presented as Mean \pm standard deviation $(n=6)$. Values not sharing a common superscript ${ }^{\dagger} \mathrm{p}<0.05$ and ${ }^{\S} \mathrm{p}<0.01$ (DMRT).

\subsection{Effect of AgNps-HEAE on lipid profile in STZ induced levels of plasma}

Table. 3 illustrations that the concentrations plasma lipids (TC, FFA, Phospholipids, TG, LDL and VLDL) were significantly increased and HDL level significantly decreased in diabetic rats as compared to the control. AgNps-HEAE orally treated with diabetic rats significantly reduced levels of TC, FFA, Phospholipids, 
TG, LDL and VLDL and HDL level significantly improved when compare to diabetic rats. There is no changes in the group of control, standard drug glibenclamide, AgNps-HEAE alone treated experimental rats.

Table. 3 Effect of AgNps-HEAE on liver marker enzymes activities in STZ induced diabetic rats of liver.

\begin{tabular}{|c|c|c|c|c|c|}
\hline Groups & Control & Diabetic rats & $\begin{array}{l}\text { Diabetic rats } \\
+\quad \text { AgNps- } \\
\text { HEAE } \\
200 \mu g / k g \text { b.w. }\end{array}$ & $\begin{array}{l}\text { Diabetic rats } \\
+\quad \text { AgNps- } \\
\text { HEAE } \\
400 \mu g / k g \text { b.w. }\end{array}$ & Glibencilamide \\
\hline $\mathrm{TC}(\mathrm{mg} / \mathrm{dl})$ & $127.67 \pm 0.42$ & $247.00 \pm 0.82^{*}$ & $172.67 \pm 0.61^{\dagger}$ & $143.00 \pm 0.52^{\S}$ & $135.67 \pm 0.84^{\S}$ \\
\hline$\overline{\mathrm{FFA}(\mathrm{mg} / \mathrm{dl})}$ & $91.09 \pm 0.56$ & $182.19 \pm 1.12^{*}$ & $137.33 \pm 0.49^{\dagger}$ & $103.83 \pm 0.87^{\S}$ & $94.00 \pm 0.58^{\S}$ \\
\hline $\mathrm{PL}(\mathrm{mg} / \mathrm{dl})$ & $118.50 \pm 0.43$ & $161.67 \pm 1.12^{*}$ & $137.67 \pm 0.42^{\dagger}$ & $124.17 \pm 0.48^{\S}$ & $120.83 \pm 0.31^{\S}$ \\
\hline $\mathrm{TG}(\mathrm{mg} / \mathrm{dl})$ & $112.83 \pm 0.60$ & $173.67 \pm 0.76^{*}$ & $147.33 \pm 0.49^{\dagger}$ & $120.33 \pm 0.33^{8}$ & $115.83 \pm 0.65^{\S}$ \\
\hline $\mathrm{HDL}(\mathrm{mg} / \mathrm{dl})$ & $55.47 \pm 0.61$ & $34.30 \pm 0.52^{*}$ & $43.25 \pm 0.31^{\dagger}$ & $50.36 \pm 0.13^{\S}$ & $53.55 \pm 0.21^{\S}$ \\
\hline $\mathrm{LDL}(\mathrm{mg} / \mathrm{dl})$ & $49.64 \pm 0.99$ & $177.97 \pm 1.29^{*}$ & $99.95 \pm 0.54^{\dagger}$ & $68.57 \pm 0.62^{\S}$ & $58.95 \pm 0.91^{\S}$ \\
\hline VLDL(mg/dl) & $22.57 \pm 0.12$ & $34.73 \pm 0.15^{*}$ & $29.47 \pm 0.10^{\dagger}$ & $24.07 \pm 0.07^{8}$ & $23.17 \pm 0.13^{\S}$ \\
\hline
\end{tabular}

Antidiabetic effect of AgNps-HEAE on lipid profile in STZ induced Diabetic Rats. Values presented as Mean \pm standard deviation $(n=6)$. Values not sharing a common superscript ${ }^{\dagger} \mathrm{p}<0.05$ and ${ }^{\S} \mathrm{p}<0.01$ (DMRT).

\subsection{Effect of AgNps-HEAE on liver marker enzymes activities in STZ induced diabetic rats of liver.}

The effect of AgNps-HEAE in the activities of liver marker enzymes like AST, ALT, ALP, GGT in control and experimental rats. The liver marker enzymes activities significantly increased in STZ induced diabetic rats (Fig. 2). Diabetic rats orally administration of AgNps-HEAE significantly reduced liver marker enzymes when compare to the diabetic rats. No changes in activities of liver marker enzymes in control, glibenclamide standard drug and AgNps-HEAE alone treated experimental rats.

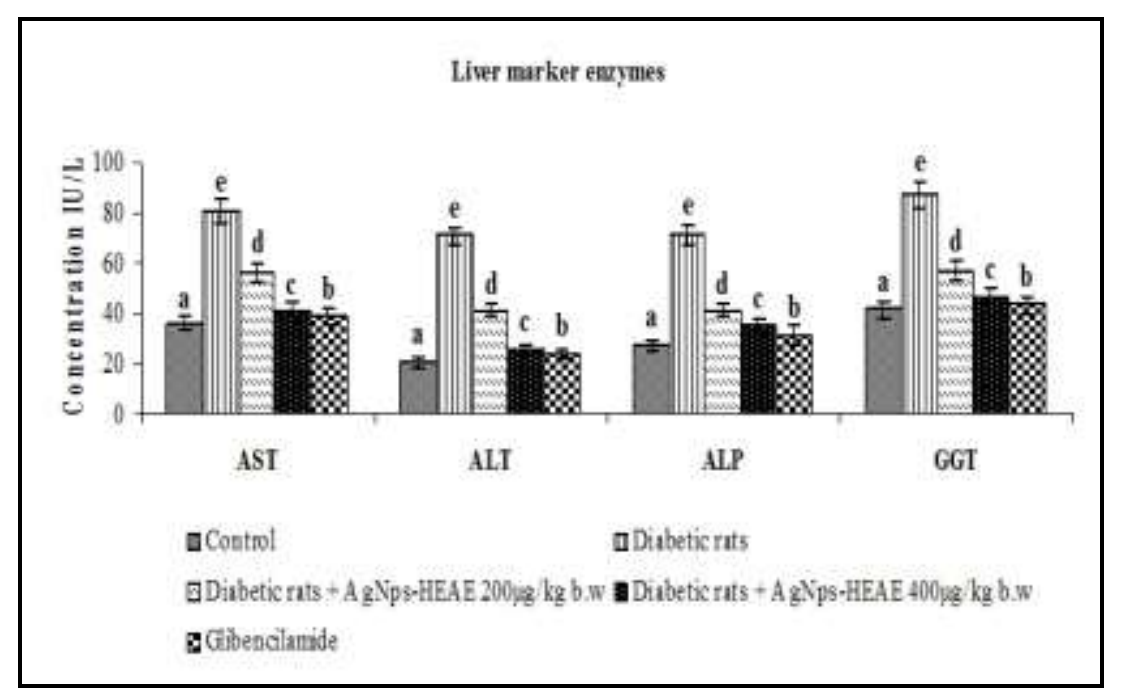

Fig 2 Liver marker enzymes

\subsection{Effect of AgNps-HEAE on urea, uric acid and creatinine in STZ induced diabetic rats of plasma}

Table 1 demonstrate that the levels of plasma urea, uric acid and creatinine in experimental rats. The urea, uric acid and creatinine levels were significantly increased in STZ induced diabetic rats. Oral administration of AgNps-HEAE to diabetic rats had significant changes in these levels to near normal. There is no changes in control, glibenclamide standard drug and AgNps-HEAE alone treated experimental rats.

\subsection{Effect of AgNps-HEAE on TBARS and HP levels of liver, kidney and pancreas}

Table $4 \& 5$ exemplify the level of TBARS and HP in plasma and tissues of control and diabetic rats. The levels of TBARS and HP were increased significantly higher than control rats, whereas diabetic rats treated with AgNps- HEAE significantly restored the altered values of TBARS and HP to near normal in the tissues of 
Nirubama Kumar /International Journal of ChemTech Research, 2018,11(03): 265-283.

liver, kidney and pancreas. There is no significant changes in control, standard drug glibencilamide and AgNpsHEAE alone treated experimental rats.

Table. 4 Effect of AgNps-HEAE on TBARS, HP and Antioxidant levels

\begin{tabular}{|c|c|c|c|c|c|}
\hline Groups & Control & $\begin{array}{l}\text { Diabetic } \\
\text { rats }\end{array}$ & 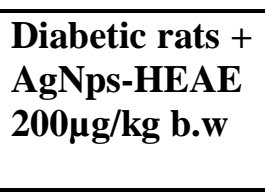 & $\begin{array}{l}\text { Diabetic rats } \\
+\quad \text { AgNps- } \\
\text { HEAE } \\
400 \mu \mathrm{g} / \mathrm{kg} \text { b.w }\end{array}$ & Glibencilamide \\
\hline \multicolumn{6}{|l|}{ Liver } \\
\hline $\begin{array}{l}\text { TBARS (nmol } \\
\text { MDA/hr/g tissue) }\end{array}$ & $2.06 \pm 0.02$ & $6.57 \pm 0.07^{*}$ & $2.88 \pm 0.02^{\S}$ & $2.55 \pm 0.03^{\S}$ & $4.25 \pm 0.06^{\dagger}$ \\
\hline $\begin{array}{l}\text { Hydroperoxide }(\mathrm{nmol} / \mathrm{g} \\
\text { tissue) }\end{array}$ & $53.83 \pm 0.79$ & $139.33 \pm 3.26^{*}$ & $70.33 \pm 0.38^{\S}$ & $61.50 \pm 0.34^{\S}$ & $139.33 \pm 3.26^{\dagger}$ \\
\hline $\begin{array}{l}\text { SOD }(\mu \mathrm{mol} / \mathrm{min} / \mathrm{mg} \\
\text { protein })\end{array}$ & $8.33 \pm 0.05$ & $4.31 \pm 0.06^{*}$ & $5.54 \pm 0.04^{\dagger}$ & $7.18 \pm 0.02^{\S}$ & $7.85 \pm 0.17^{\S}$ \\
\hline $\begin{array}{l}\mathrm{CAT}(\mu \mathrm{mol} / \mathrm{min} / \mathrm{mg} \\
\text { protein) }\end{array}$ & $72.16 \pm 0.50$ & $42.55 \pm 0.17^{*}$ & $54.55 \pm 0.17^{\dagger}$ & $69.67 \pm 0.15^{\S}$ & $71.05 \pm 0.25^{\S}$ \\
\hline $\begin{array}{l}\text { GPx }(\mathrm{mol} / \mathrm{min} / \mathrm{mg} \\
\text { protein) }\end{array}$ & $6.67 \pm 0.04$ & $3.18 \pm 0.02^{*}$ & $4.32 \pm 0.05^{\dagger}$ & $5.68 \pm 0.06^{\S}$ & $6.09 \pm 0.02^{\S}$ \\
\hline $\begin{array}{l}\mathrm{GR}(\mathrm{nmol} / \mathrm{min} / \mathrm{mg} \\
\text { protein) }\end{array}$ & $3.25 \pm 0.02$ & $0.44 \pm 0.02^{*}$ & $1.34 \pm 0.02^{\dagger}$ & $2.85 \pm 0.04^{\S}$ & $3.04 \pm 0.03^{\S}$ \\
\hline $\begin{array}{l}\text { GST }(\mu \mathrm{mol} / \mathrm{min} / \mathrm{mg} \\
\text { protein })\end{array}$ & $0.80 \pm 0.04$ & $0.25 \pm 0.00^{*}$ & $0.53 \pm 0.06^{\dagger}$ & $0.69 \pm 0.01^{\S}$ & $0.74 \pm 0.00^{\S}$ \\
\hline GSH $(\mu \mathrm{mol} / \mathrm{g} / \mathrm{tissue})$ & $23.82 \pm 0.26$ & $12.35 \pm 0.08^{*}$ & $16.80 \pm 0.21^{\dagger}$ & $20.35 \pm 0.08^{\S}$ & $21.93 \pm 0.14^{\S}$ \\
\hline Vitamin $C(\mu \mathrm{g} / \mathrm{g}$ tissue $)$ & $235.58 \pm 0.48$ & $188.66 \pm 0.23^{*}$ & $205.30 \pm 0.32^{\dagger}$ & $226.82 \pm 0.61^{\S}$ & $228.74 \pm 0.28^{\S}$ \\
\hline Vitamin $\mathrm{E}(\mu \mathrm{g} / \mathrm{g}$ tissue $)$ & $20.54 \pm 0.10$ & $9.40 \pm 0.03^{*}$ & $13.44 \pm 0.04$ & $18.54 \pm 0.06^{\S}$ & $18.46 \pm 0.07^{\S}$ \\
\hline \multicolumn{6}{|l|}{ Kidney } \\
\hline $\begin{array}{l}\text { TBARS (nmol } \\
\text { MDA/hr/g tissue) }\end{array}$ & $3.73 \pm 0.05$ & $7.45 \pm 0.06^{*}$ & $4.26 \pm 0.03^{\S}$ & $4.10 \pm 0.02^{\S}$ & $5.77 \pm 0.05^{\dagger}$ \\
\hline $\begin{array}{l}\text { Hydroperoxide }(\mathrm{nmol} / \mathrm{g} \\
\text { tissue) }\end{array}$ & $43.33 \pm 0.33$ & $112.67 \pm 1.02^{*}$ & $55.33 \pm 0.67^{\S}$ & $52.83 \pm 0.60^{\S}$ & $70.33 \pm 0.42^{\dagger}$ \\
\hline $\begin{array}{l}\text { SOD }(\mu \mathrm{mol} / \mathrm{min} / \mathrm{mg} \\
\text { protein })\end{array}$ & $13.33 \pm 0.05$ & $6.22 \pm 0.03^{*}$ & $9.22 \pm 0.05^{\dagger}$ & $11.97 \pm 0.06^{\S}$ & $12.39 \pm 0.06^{\S}$ \\
\hline $\begin{array}{l}\mathrm{CAT}(\mu \mathrm{mol} / \mathrm{min} / \mathrm{mg} \\
\text { protein) }\end{array}$ & $54.40 \pm 0.27$ & $33.59 \pm 0.25^{*}$ & $44.16 \pm 0.16^{\dagger}$ & $51.92 \pm 0.43^{\S}$ & $53.94 \pm 0.35^{\S}$ \\
\hline $\begin{array}{l}\text { GPx }(\mathrm{mol} / \mathrm{min} / \mathrm{mg} \\
\text { protein) }\end{array}$ & $5.70 \pm 0.06$ & $2.20 \pm 0.05^{*}$ & $3.77 \pm 0.03^{\dagger}$ & $5.06 \pm 0.01^{\S}$ & $5.10 \pm 0.04^{\S}$ \\
\hline $\begin{array}{l}\mathrm{GR}(\mathrm{nmol} / \mathrm{min} / \mathrm{mg} \\
\text { protein) }\end{array}$ & $1.78 \pm 0.03$ & $0.33 \pm 0.01^{*}$ & $0.82 \pm 0.03^{\dagger}$ & $1.45 \pm 0.02^{\S}$ & $1.54 \pm 0.03^{\S}$ \\
\hline $\begin{array}{l}\mathrm{GST}(\mu \mathrm{mol} / \mathrm{min} / \mathrm{mg} \\
\text { protein })\end{array}$ & $0.57 \pm 0.02$ & $0.12 \pm 0.01^{*}$ & $0.30 \pm 0.01^{\dagger}$ & $0.47 \pm 0.01^{\S}$ & $0.52 \pm 0.01^{\S}$ \\
\hline GSH $(\mu \mathrm{mol} / \mathrm{g} / \mathrm{tissue})$ & $15.21 \pm 0.20$ & $7.25 \pm 0.03^{*}$ & $10.74 \pm 0.19^{\dagger}$ & $14.91 \pm 0.04^{\S}$ & $15.06 \pm 0.01^{\S}$ \\
\hline Vitamin $C(\mu \mathrm{g} / \mathrm{g}$ tissue $)$ & $188.47 \pm 0.38$ & $146.92 \pm 0.18$ & $162.24 \pm 0.26^{\dagger}$ & $181.46 \pm 0.49^{\S}$ & $182.99 \pm 0.22^{\S}$ \\
\hline Vitamin $\mathrm{E}(\mu \mathrm{g} / \mathrm{g}$ tissue $)$ & $16.43 \pm 0.08$ & $7.42 \pm 0.03^{*}$ & $11.65 \pm 0.03^{\dagger}$ & $14.83 \pm 0.05^{\S}$ & $14.77 \pm 0.06^{\S}$ \\
\hline
\end{tabular}

Antidiabetic effect of AgNps-HEAE on various biochemical parameters in STZ induced Diabetic Rats. Values presented as Mean \pm standard deviation $(n=6)$. Values not sharing a common superscript ${ }^{\dagger} p<0.05$ and ${ }^{\S} \mathrm{p}<0.01$ (DMRT).

\subsection{Activities of AgNps-HEAE on enzymatic antioxidant of liver, kidney and pancreas}

The activities of enzymatic antioxidants namely SOD, CAT, GPx, GST and GR in the liver, kidney and pancreas of control and experimental rats are given in Table 4 \& 5. The activities of enzymatic antioxidants 
namely SOD, CAT, GPx, GST and GR in the liver, kidney and pancreas were significantly decreased in diabetic rats when compared with control rats. Diabetic rats treated with AgNps - HEAE showed significant increase in the activities of enzymatic antioxidants, which reflects the restoration of antioxidant enzymes system to near normal. There is no significant changes in control, standard drug glibencilamide and AgNps - HEAE alone treated experimental rats.

Table. 5 Activities of AgNps-HEAE on enzymatic antioxidant of liver, kidney and pancreas

\begin{tabular}{|c|c|c|c|c|c|}
\hline Groups & Control & $\begin{array}{l}\text { Diabetic } \\
\text { rats }\end{array}$ & $\begin{array}{l}\text { Diabetic rats } \\
+\quad \text { AgNps- } \\
\text { HEAE } \\
200 \mu \mathrm{g} / \mathrm{kg} \text { b.w }\end{array}$ & $\begin{array}{l}\text { Diabetic rats } \\
+\quad \text { AgNps- } \\
\text { HEAE } \\
400 \mu g / k g \text { b.w }\end{array}$ & Glibencilamide \\
\hline \multicolumn{6}{|l|}{ Pancreas } \\
\hline $\begin{array}{l}\text { TBARS (nmol } \\
\text { MDA/hr/g tissue) }\end{array}$ & $0.64 \pm 0.01$ & $3.17 \pm 0.08^{*}$ & $0.86 \pm 0.01^{\S}$ & $0.78 \pm 0.14^{\S}$ & $1.88 \pm 0.03^{\dagger}$ \\
\hline $\begin{array}{l}\text { Hydroperoxide (nmol/g } \\
\text { tissue) }\end{array}$ & $15.50 \pm 0.43$ & $54.83 \pm 0.70^{*}$ & $19.14 \pm 0.03^{\S}$ & $18.23 \pm 0.04^{\S}$ & $32.77 \pm 0.51^{\dagger}$ \\
\hline $\begin{array}{l}\mathrm{SOD}(\mu \mathrm{mol} / \mathrm{min} / \mathrm{mg} \\
\text { protein })\end{array}$ & $11.29 \pm 0.05$ & $6.15 \pm 0.02^{*}$ & $8.76 \pm 0.06$ & $10.05 \pm 0.05^{\S}$ & $10.44 \pm 0.05^{\S}$ \\
\hline $\begin{array}{l}\text { CAT }(\mu \mathrm{mol} / \mathrm{min} / \mathrm{mg} \\
\text { protein) }\end{array}$ & $36.10 \pm 0.32$ & $23.95 \pm 0.17^{*}$ & $29.56 \pm 0.13^{\dagger}$ & $32.99 \pm 0.07^{\S}$ & $33.74 \pm 0.13^{\S}$ \\
\hline $\begin{array}{l}\text { GPx }(\mathrm{mol} / \mathrm{min} / \mathrm{mg} \\
\text { protein) }\end{array}$ & $1.44 \pm 0.06$ & $0.51 \pm 0.03^{*}$ & $0.76 \pm 0.03^{\dagger}$ & $1.09 \pm 0.03^{\S}$ & $1.16 \pm 0.03^{\S}$ \\
\hline $\begin{array}{l}\mathrm{GR}(\mathrm{nmol} / \mathrm{min} / \mathrm{mg} \\
\text { protein) }\end{array}$ & $3.68 \pm 0.05$ & $1.16 \pm 0.02^{*}$ & $2.06 \pm 0.02^{\dagger}$ & $3.01 \pm 0.03^{\S}$ & $3.11 \pm 0.03^{\S}$ \\
\hline $\begin{array}{l}\text { GST }(\mu \mathrm{mol} / \mathrm{min} / \mathrm{mg} \\
\text { protein) }\end{array}$ & $0.51 \pm 0.01$ & $0.08 \pm 0.00^{*}$ & $0.23 \pm 0.01^{\dagger}$ & $0.40 \pm 0.01^{\S}$ & $0.47 \pm 0.01^{\S}$ \\
\hline GSH $(\mu \mathrm{mol} / \mathrm{g} /$ tissue $)$ & $6.58 \pm 0.09$ & $2.33 \pm 0.08^{*}$ & $4.21 \pm 0.03^{\dagger}$ & $6.01 \pm 0.04^{\S}$ & $6.16 \pm 0.02^{\S}$ \\
\hline Vitamin $C(\mu \mathrm{g} / \mathrm{g}$ tissue $)$ & $110.47 \pm 0.38$ & $88.92 \pm 0.18^{*}$ & $94.24 \pm 0.26^{\dagger}$ & $103.46 \pm 0.49^{\S}$ & $104.99 \pm 0.22^{\S}$ \\
\hline Vitamin $\mathrm{E}(\mu \mathrm{g} / \mathrm{g}$ tissue $)$ & $12.61 \pm 0.08$ & $3.60 \pm 0.03^{*}$ & $7.83 \pm 0.03^{\dagger}$ & $11.01 \pm 0.05^{\S}$ & $10.95 \pm 0.06^{\S}$ \\
\hline
\end{tabular}

Antidiabetic effect of AgNps-HEAE on various biochemical parameters in STZ induced Diabetic Rats. Values presented as Mean \pm standard deviation $(n=6)$. Values not sharing a common superscript ${ }^{\dagger} p<0.05$ and ${ }^{\S} p<0.01$ (DMRT).

\subsection{Activities of AgNps-HEAE on non-enzymatic antioxidant of liver, kidney and pancreas}

The levels of non-enzymatic antioxidants, namely GSH, vitamin $\mathrm{C}$ and vitamin $\mathrm{E}$ in control and diabetic rats are given in Table $4 \& 5$. There was a significantly decreased in the level of GSH, vitamin $\mathrm{C}$ and vitamin $\mathrm{E}$ in diabetic rats. Administration of AgNps-HEAE to diabetic rats exhibited a significantly increased in the levels of these non-enzymatic antioxidants and there is no significant changes in control, standard drug glibencilamide and AgNps - HEAE alone treated experimental rats.

\subsection{Effect of AgNps-HEAE on histopathology characterization of liver, kidney and pancreas}

Fig.3a represents the microphotographs of H\&E staining of hepatic and renal tissues of control and experimental rats. Pathological changes of STZ-induced diabetic rat liver include congestion of sinusoidal dilatation, inflammation of the central vein and focal necrosis in the hepatocytes in diabetic control rats. The above pathological changes were reduced in rats treated with AgNps-HEAE experimental rats.

Fig. 3b illustrations the diabetic control rat kidney showed tubular epithelial degeneration with mononuclear cell infiltrated edema and necrosis structures and diabetic rats treated with AgNps- HEAE did not show any tissue damage or neuronal alterations, although it shows mild degeneration of epithelial cells with mild necrosis.

Fig. 3c showed multiple foci of hemorrhage, necrosis and swelling of tubules in diabetic rats. These changes were reduced in AgNps- HEAE treated rats. No histological alterations were observed in liver, kidney and pancreas of control rats. There is no histological change in standard drug glibencilamide and AgNps HEAE alone treated experimental rats of liver, kidney and pancreas. 
For all the parameters studied, AgNps-HEAE at a dose of $400 \mu \mathrm{g} / \mathrm{kg}$ b.w. showed significant better effect than $200 \mu \mathrm{g} / \mathrm{kg}$ b.w. based on these data, the protective effect of dose was fixed at $400 \mu \mathrm{g} / \mathrm{kg}$ b.w. Hence, further studies were carried out using this current dose.

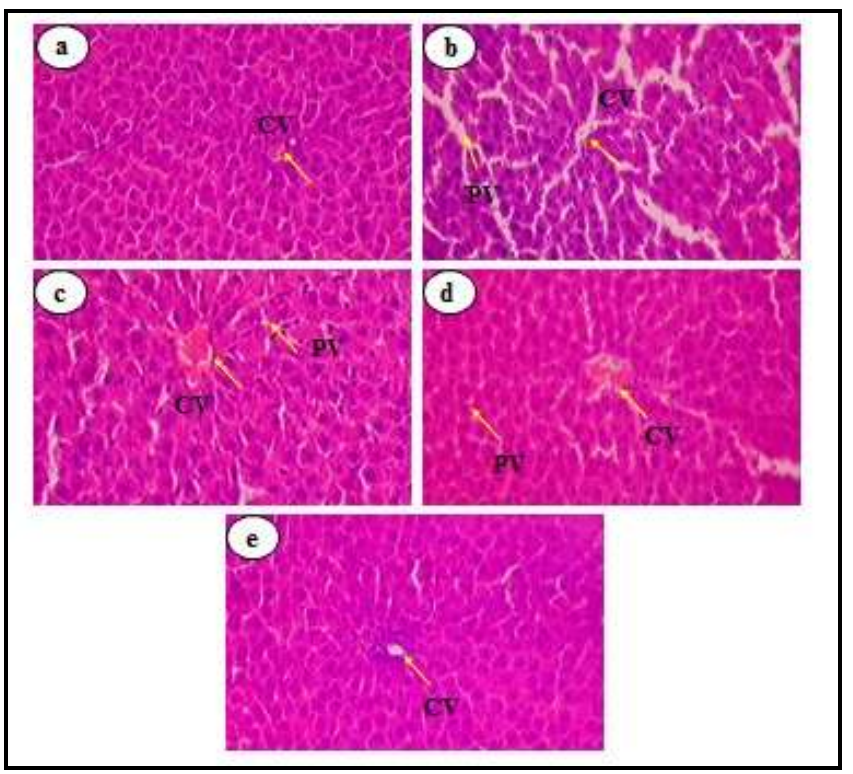

Fig 3a Histopathology of liver

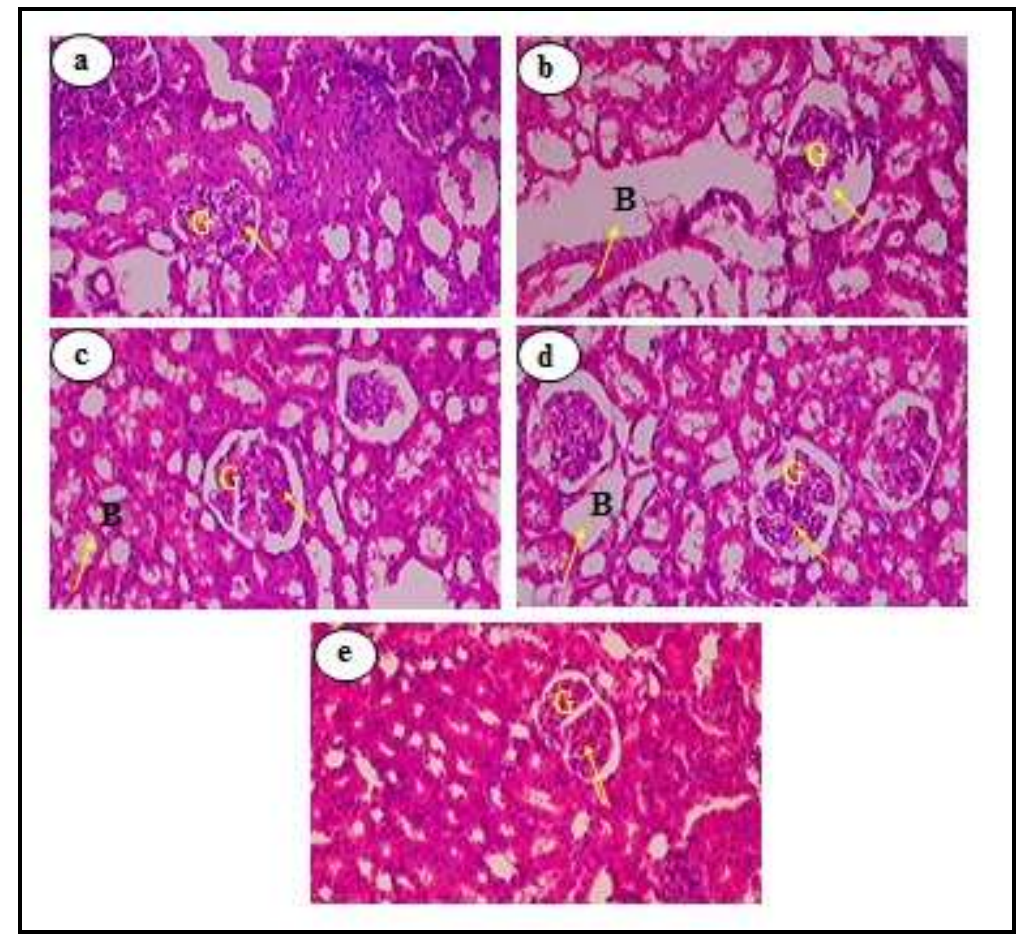

Fig 3b Histopathology of Kidney 


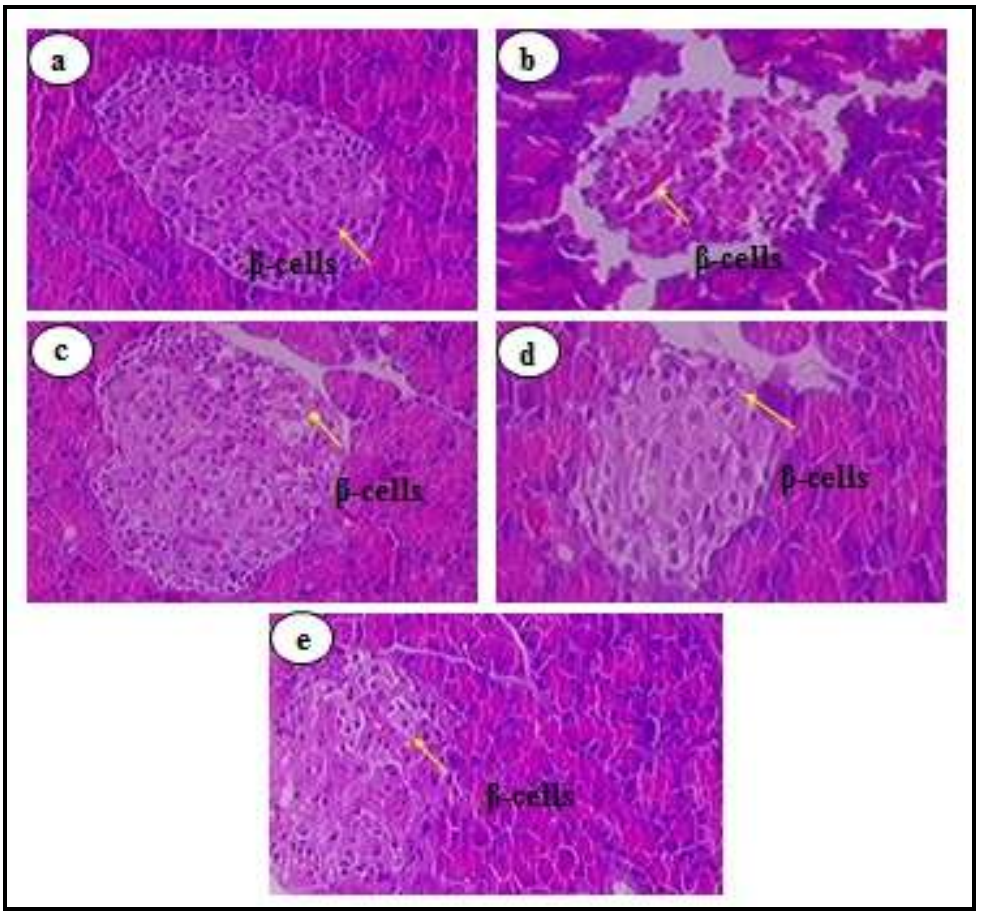

Fig 3C Histopathology of Pancreas

\section{Discussion}

Diabetes mellitus is a group of metabolic disease characterized by disarrangements in carbohydrate, proteins and fat metabolism caused by deficiency of circulating insulin levels. In the present study, diabetic rats reveal a significant increase in plasma glucose level. This result is in stable with other studies in rats. The increased glucose level might be due to the fact that STZ causes significant reduction in insulin release by the damage of pancreatic $\beta$-cells.

Experimental animal models have been recommended to be one of the best ways to know the pathophysiology of any disease (Chatzigeorgiou et al., 2009; Ali et al., 2011). In this study, the intra-peritoneal administration of streptozotocin (STZ) effectively induced diabetes mellitus in wistar rats which was confirmed by elevated levels of fasting blood glucose, three days after STZ injection. This agrees with the reports of Mohammed et al. (2008), and Krishna et al. (2012).

Several studies confirmed that a variety of plant extracts effectively lowered the glucose level in STZ induced diabetic rats. We have noticed a significant decrease in glucose level in AgNPS-HEAE treated diabetic rats when compared with control group. The possible mechanism of hypoglycemic action may be through potential of pancreatic excretion of insulin from beta cells of islets or due to increased transport of blood glucose to the peripheral tissue. The amount of insulin level has been revealed to be a important key of insulin secretion rather than insulin alone.[60] Insulin level was significantly decreased in STZ-induced diabetic rats due to the damage of $\beta$-cells of pancreas there by inhibit insulin release.

AgNPS-HEAE plant had a more articulate effect at a high dose than when administered at a low dose. This agrees with the report of Bamidele et al (2014), that AgNPS-HEAE treated gruops significantly reduced blood glucose level in STZ induced diabetic wistar rats. The possible mechanism involved in the anti hyperglycaemic action of AgNPS-HEAE may be due to reduction of nitric oxide (NO) production of reactive oxygen species (ROS) (Kim et al., 2007) due to the presence of anti oxidants presence in the plant (Olajire and Azeez, 2011) which are known to scavenge the free radicals produced by oxidative damage in the disease state (Bamidele et al., 2010; Nirmala et al., 2011) or decrease blood glucose through inhibition of STZ-induced peroxidation and apoptosis in beta cells by increasing glycogen content in liver and muscle; and stimulate glucokinase, hexokinase and phosphor fructokinase (Rathi et al., 2002).

The decreased level of total haemoglobin observed in diabetic rats might be due to the increased formation of glycosylated haemoglobin. Glycosylated haemoglobin was found to increase in diabetes. [61] The 
amount of glycosylated haemoglobin remains the standard biochemical marker for the estimation of glycemic control in patients with diabetes. [62] The excess amount of glucose in the blood reacts with haemoglobin to form glycosylated haemoglobin in diabetes mellitus. Oral administration of AgNps-HEAE to STZ-induced diabetic rats reduced the formation of glycosylated haemoglobin by advantage of its normoglycemic activity. Since the level of glycosylated haemoglobin has been revealed to provide an index of blood glucose concentration, the decreased level of glycosylated haemoglobin and the increased level of haemoglobin in treated diabetic rats exhibit the anti hyperglycemic activity of AgNps-HEAE.

Total protein was also decreased in serum of STZ induced diabetic rats. The decreased level of total protein is due to an increased conversion of glycogenic amino acid to carbon dioxide and water. [63]. Diabetic treated with AgNPs-HEAE shows the protein level near to normal. Similarly it was also noticed in the standard drug glibencilamide treated rats.

The glucokinase activity was decreased in serum of STZ induced diabetic rats. This may be due to insulin insufficiency. The AgNps-HEAE increased the activity of glucokinase in the liver. AgNps-HEAE treated groups may stimulate insulin secretion, which activates glucokinase, thereby enhances the consumption of glucose and decreased in blood glucose levels.

The hepatic gluconeogenic enzymes i.e. glucose-6-phosphatase and fructose-1,6-bisphosphatase were significantly increased in the liver of diabetic rats [64], may be due to deficiency of insulin increase the activities of gluconeogenic enzymes resulting endogenous glucose production may be contributing to the increased glucose liberated from the liver [65]. Diabetic rats treated with AgNps-HEAE inhibited the activities of these enzymes; either may be manage of metabolic activation or inhibition of glycolysis and gluconeogenesis. These results consecutively proved that AgNps-HEAE normalizes the disturbed glucose metabolism by decreasing hepatic glucose production through insulin liberate. The glibenclamide treated rats exhibit similar more result than the extract treated rats. The enhanced actions of gluconeogenic enzymes were revealed to be reduced after treatment with other medicinal plants [66], in experimental diabetic animals and or outcome results are similar with these reports.

The Glucose 6 phosphate dehydrogenase was significantly decreased in diabetic rats and AgNps-HEAE treated diabetic rats in comparing with control group, and significantly increased in treatment with AgNpsHEAE comparing to with diabetic group. The decrease of the activity of glucose 6 phosphate dehydrogenase in STZ-induced diabetic rats was steady with other research on glucose 6 phosphate dehydrogenate [67].

Thus, stored fatty acid in the plasma produced by the STZ-induced diabetes develops the conversion of excess fatty acids into phospholipids and cholesterol in the liver. These two substances, along with excess triglycerides formed in the liver, may be out into the blood in the form of lipoproteins [68]. The observed increase in plasma phospholipids is an effect of elevated lipoproteins. Therefore, the notable hyperlipidaemia that show the diabetic state may be noticed as a result of the uninhibited activities of lipolytic hormones on fat stores. However treatment with AgNps-HEAE regulates plasma lipid status, which was most possibly moderate by a regulation of lipid metabolism.

Results of the study exhibit that the lipid profile markers such as total cholesterol, triacylglycerol and LDL-cholesterol concentrations were noticed to be higher except for the HDL-cholesterol notice which was reduced in the diabetic control group. It is well known that in uncontrolled diabetes mellitus, there will be an increase in total cholesterol, triacylglycerol and LDL-C with a related decrease in the HDL-C which contributes to coronary artery disease (Arvind et al., 2002; Selvan et al., 2008). This could be credit to abnormality in lipid metabolism due to diabetes-induced hypertriglyceridaemia and hypercholesterolaemia (Mitra et al., 1995). The increase in blood cholesterol and triacylglycerol concentrations may be due to the action of hormone sensitive lipase, which build up lipolysis and consequently increases the level of free fatty-acids and triacylglycerol in circulation. The free fatty acids are catabolized to acetyl-CoA which is further channelled to cholesterol synthesis; thus, increasing blood cholesterol level (Oyedepo, 2012).

This could be due to increased breakdown of the cholesterol in the liver, and decreased absorption of cholesterol via the chylomicrons due to inhibition of $\alpha$-glucosidase enzymes. The above result recommended that the administration of AgNPs-HEAE may improve lipid dysfunction and hence hamper the development of diabetic complications. This could be qualified to their promotion of consumption of glucose and hence depressed mobilization of fats. 
The reduction of AST and ALT activities by the extract is an indication of repair of tissue damage induced by diabetes complications. This is in agreement with [70], who found that serum transaminases returned to normal activities with the healing of tissue parenchyma and regeneration of hepatocytes and renal tissues [71]. Serum ALP is a sensitive detector for intra hepatic and extra hepatic bile obstruction. More over Alanine aminotransferase, Aspartate aminotrasnferase, Alkaline phosphatase and Gama glutamyl transaminase major enzymes for liver function and integrity. The increased level of Alanine aminotransferase, Aspartate aminotrasnferase, Alkaline phosphatase and Gama glutamyl transaminase are observed in diabetic control.

It was also observed the significant reduction of the Alanine aminotransferase, Aspartate aminotrasnferase, Alkaline phosphatase and Gama glutamyl transaminase level in AgNPS -HEAE treated and standard Glibencilamide treated diabetic rats. Some of the harmful effects of diabetes mellitus may release to hepatic dysfunction and may be corrected after use AgNPS -HEAE extract diabetic rats. Our results showed that liver function tests such as Alanine aminotransferase, Aspartate aminotrasnferase, Alkaline phosphatase and Gama glutamyl transaminase after diabetic induction and are modified after treatment which indicate that AgNPS -HEAE extract have potential of liver damage recovering effects. These results are in agreement with those of (Rawi et al., 1998), [72] who establish that the decrease of transaminases activities with treatment may be credit to enhanced liver function with the return of gluconeogensis towards its normal rate.

Moreover, a separate study by Srivastava et al. has also confirmed the potential of AgNPs to affect the activity of transaminase enzymes. In previous studies, the potential of silver nanoparticles to modulate enzyme activity was available to their affinity for thiol groups. It is possible that thiol groups in the enzymes made them attractive to the AgNPs leading to development of complexes and consequent modulation of enzyme activity.

The earlier study showed that there was an increase in serum urea and serum creatinine levels in streptozotocin induced diabetic rats. However after 21 days administration of ethanolic extract of Triumfetta pilosa has led to a significant fall in serum urea and serum creatinine levels when compared with the standard group [73]. The decreased level of AgNps - hydroalcoholic extract of A. echioides was administered at 200 and $400 \mu \mathrm{g} / \mathrm{kg}$ b.w., compared to the diabetic control rats. The standard drug (Glibenclamide) treated rats also showed a significant of the diabetic hyperglycemia induces elevation of the serum levels of urea, uric acid and creatinine, which were examine as important markers of renal function [74]. The diabetic hyperglycemia induces elevation of the plasma levels of urea, uric acid and creatinine which are significant markers of renal dysfunction and reflecting a decreased in the glomerular filtration rate were notably recovered by AgNPs HEAE. Uric acid is a product of purine metabolism. The increase in uric acid could be due to the fact that filtered uric acid is both reabsorbed and excreted in the proximal tubule through a voltage-sensitive urate channel and a urate-anion exchange mechanism. Hyperuricemia can be a result of either increased production or decreased excretion [75]. The AgNps-HEAE restored the elevated uric acid level in diabetic rats.

Hyperglycemia is associated with formation of reactive oxygen species (ROS) which causes damage particularly to liver, kidney and also pancreas. The level of lipid peroxidation (TBARS) and reactive oxygen species (superoxide anion, hydrogen peroxide $\left(\mathrm{H}_{2} \mathrm{O}_{2}\right)$, and hydroxyl radical) are common markers of oxidative stress in STZ induced diabetic rats. Lipid peroxidation refers to the oxidative degradation of lipids that impairs cell membrane functions resulting in cell damage and leading to several pathologies and cytotoxicity. Malondialdehyde (MDA) is one of the end products of lipid peroxidation which is usually measured as a marker for oxidative stress [76]. Expected with these results, we found that decreased levels of TBARS and Hydroperoxide in AgNPs - HEAE treated diabetic experimental rats. It shows the potential of antioxidant capacity.

The previous studies have also revealed that there is a close relationship between the increase of free radicals, blood glucose and lipid peroxidation (LPO) in the evolution of diabetes [77]. In addition, aldehydes produced from the decomposition of PUFAs are capable of forming cross-linking in proteins and nucleic acid, which leads to inactivation of many cellular constituents, membranes and enzymes [78]. In this study, the concentrations of MDA decreased after treating with AgNPs - HEAE, it shows the scavenging of free radicals.

The increase in blood glucose level might be due to lack of insulin as a result of damage of pancreas intermediated by STZ action. STZ boosts ATP dephosphorylation, which in turn generates superoxide anions, hydrogen peroxide, and hydroxyl radicals. This leads to an increase in the intracellular peroxides in pancreatic islets, which may induce damage due to the reactive oxygen species (ROS) (Lenzen et al., 1996.) [79] Under hyperglycaemic conditions, antioxidants are supposed to regenerate damaged extracellular matrix proteins and 
promote cell growth (Smith et al., 1992). [80] Thus, antioxidants might help in the administration of diabetes mellitus.

Antioxidant systems build up the body's defence system that plays an essential role in scavenging free radicals, thereby preventive the toxicity of reactive oxygen species (ROS). ROS are neutralized by a battery of antioxidants, which can be classified into two types: enzymatic (e.g.: superoxide dismutase SOD, catalase CAT and glutathione peroxidase GPx) and non-enzymatic systems (e.g.: glutathione GSH, vitamins A, C and E) [81].

Superoxide dismutase, a metalloprotein, is primarily involved in the antioxidant defense by scavenging the superoxide radicals. In hyperglycaemia, glucose undergoes auto-oxidation and produces superoxide radicals which lead to lipid peroxidation [82]. Catalase, is an enzyme, it catalyzes the decomposition of hydrogen peroxide and protecting the cell from oxidative damage by reactive oxygen species. [83].

Enzymatic antioxidants such as SOD and catalase are examined primary enzymes since they are involved in the suppressed of ROS superoxide dismutase is the antioxidant of defence against neutralizing the free radicals. The function of catalyse is to conversion of superoxide radicals to hydrogen peroxide and reduces the toxic effects due to this radical or other free radicals derived from secondary reaction. The hydrogen peroxide produced by SOD is excreted as water, based on the activity of GSH-Px and catalase, thereby protecting the body from oxygen toxicity [84]. Hence in the present study activities of SOD, catalase and GSHPx are increased in the treatment with AgNPs - HEAE.

GPx detoxifies hydrogen peroxide to water through the oxidation of reduced glutathione [85]. The decreased level of GPx activity in STZ induced diabetic control rats indicates an important adaptive response to increased peroxidative stress. The administration of AgNps-HEAE showed increased the formation of GPx activity when compared to diabetic control rats. The ability of AgNps-HEAE to restore the antioxidant status in diabetic rats clearly indicates its neutralizing the ability of free radicals.

Reduced glutathione is an important bio molecule for the removal of reactive intermediates by reduction of hydroperoxidase in the presence of glutathione peroxidase. GSH also functions as a free radical quenching and helps to repair free radical mediated biological damage. [86]

Glutathione peroxidase, with its peroxidase activity protect the oxidative damage, it catalyses the reaction to reduced lipid hydroperoxides. These antioxidants interact and synergistically stabilize, or deactivate, free radicals before they attack cells. Glutathione and vitamin $\mathrm{C}$ work related to neutralize free radicals and have a potential of antioxidants. Our result showed that increased level of GR and GST in diabetic rats and orally administrated of AgNPs - HEAE treated with diabetic rats levels increased.

Craven et al. stated that the increasing of glycemic control done by vitamin $\mathrm{C}$ was mainly initiated by a good effect of this antioxidant on $\beta$ cells. Yet there may be a possibility that the antioxidant treatment could have exerted an influence on target tissues other than the $\beta$ cells such as muscle and fat. It is postulated that the antioxidant treatment has positive effects on preservation of $\beta$ cell function in the diabetic patients, even though the effects may not be exerted totally through its direct action on $\beta$ cells. Also, the influence on insulin sensitivity, the antioxidant treatment indeed reduced blood glucose level. [87] The inhibition of protein glycosylation by Vitamin $\mathrm{C}$ and Vitamin $\mathrm{E}$ appears to be one of the possibilities. In our result finding that decreased level of vitamin $\mathrm{A}$, vitamin $\mathrm{C}$ and vitamin $\mathrm{E}$ in diabetic rats and treatment with administration of AgNPs - HEAE shows the potential of antioxidant properties.

The promising potential of silver/gold nanoparticles in treating inflammatory and auto immune diseases [88] have amplify greater interest to investigate the antihyperglycemic activity of the nano particles in the diabetic system. Another study [89] conducted in vivo (in mice) experiments, suggested the efficacy of encapsulated nano Syzygium jambolanum to have localized in the brain tissue of mice which recommended that it could efficiently cross the blood brain barrier, especially the hypothalamic region, proposed to be the glucose sensor region, which plays a critical role in initiating the counter regulatory response to glucose homeostasis. The overall results suggested that AgNps-HEAE had a greater potential by itself, indicating its possible use in the future drug design and management of Diabetes meilitus.

Afifi et al. stated that $\mathrm{ZnONPs}$ were able to decrease the oxidative stress in testicular tissue as manifested from decreased MDA levels, higher GSH amount, and induced antioxidant enzymes (SOD, CAT, 
GPx, GR, and GST) activities and gene expressions in the testicular tissue of diabetic rats. We proved the antioxidant ability of ZnONPs in diabetic rat brain tissue.

The liver, kidney and pancreas samples of STZ- induced rats showed that the central veins are dilated and congested and the hepatocytes show peripheral fatty changes [90]. The histopathological pattern of the livers of the rats co-administrated with AgNps - HEAE showed almost normal architecture of liver, kidney and Pancreas shows with few fatty changes.

The antioxidant principles from herbal sources are involved in their effects and provides an huge scope in correcting the imbalance triggered during free radical damage, followed by the positive uphold of antioxidant status in AgNps - HEAE further the enzymatic antioxidants a positive indication in restoring the histopathological study validates the effectiveness of AgNPs - hydroalcholoic extract of A. echioides in normalizing the anti-diabetic architecture.

\section{Conclusion}

In conclusion, the findings of this study support this view that medicinal plant A. echioides is a promising source of potential antioxidant and antidiabetic principles and may be efficient as preventive agents in the pathogenesis of some diseases. AgNPs - hydroalcholoic extract of A. echioides showed higher inhibition rates against free radicals and excellent antioxidant and antidiabetic properties. This may be a good natural antioxidant and antidiabetic alternative to existing synthetic antioxidant in the food and medicinal industries. Hence it is necessary to indicate the A.echioides and further analysis is recommended for future research is required which may lead to development for drug formulation.

\section{Abbreviations:}

A. echioides - Andrographis echioides

STZ - Streptozotocin

AgNPs - Silver nanoparticle

DM - Diabetes mellitus

HEAE - Hydroalcholic exract of Andrographis echioides

SOD - Superoxide dismutase

GPx - Glutathione peroxidase

AST - Aspartate transaminase

ALP - Alkaline phosphatise

ALT - Alanine transaminase

GGT - Gamma glutamyl transpeptidase

CPCSEA - Committee for the Purpose of Control and Supervision of Experiments on Animals

\section{References}

1. Jing Li, Fayong Gong, Fenglin L, Hypoglycemic and hypolipidemic effects of flavonoids from tatary buck wheat in type 2 diabetic rats. Biomedical Research, 27 (2016) 132-137.

2. H.King, R.E. Aubert, and W.H. Herman, Global burden of diabetes 1995 -2025:Prevalence, numerical estimates, and projections. Diabetes Care, 21(1998) 1414 -1431.

3. K. Subramani, S. Pathak, H. Hosseinkhani, Dig. J. Nanomater. Bios. 7, 85 (2012).

4. M.E Dolan, Inhibition of DNA repair as a means of increasing the antitumor activity of DNA active agents. Adv Drug Del Rev. (1997) 26 105-118.

5. A. Junod, A.E. Lambert, L. Orci, R.Pictet, A.E. Gonet, R. Enold AE Studies of the diabetogenic action of streptozotocin. Proc Soc Exp Biol Med. (1967) 126 201-205.

6. C.R. Kulkarni, M.M. Joglekar, S.B. Patil, A.U. Arvindekar, Antihyperglycemic and antihyperlipidemic effect of Santalum album in streptozotocin induced diabetic rats, Pharm. Biol. 50 (2012) 360-365.

7. D.J. Betteridge, LDL heterogeneity: implications for atherogenicity in insulin resistance and NIDDM. Diabetologia 40 (1997) 149-151.

8. G.N. Levinthal, A.J. Tavill . Liver disease and Diabetes Mellitus. Clin. Diabetes. (1999) 17 73-75.

9. G. Marchesini , M. Brizi , G.Bianchi, Nonalcoholic fatty liver disease. A feature of metabolic syndrome. Diabetes. (2001) 50 1844-1850. 
10. N. Sattar, O. Scherbakova, I. Ford, D.S. O'Reilly, A. Stanley,E. Forrest E, P.W. Macfarlane, C.J. Packard, S.M .Cobbe, J. Shepherd, Elevated alanine aminotransferase predicts new-onset type 2 diabetes independently of classical risk factors, metabolic syndrome, and C-reactive protein in the West of Scotland coronary prevention study. Diabetes. (2004) 53 2855-2860.

11. S. Yamagishi, T. Matsui , Nitric oxide, a Janus-faced therapeutic target for diabetic microangiopathyPharmacol Res. (2011) 64 187-194.

12. T. Offer, T. Russo, Samuni, A, The proxidative activity of SOD and nitro oxide SOD mimes. FASEB $14,(2000) 1215$.

13. M. Ramanathan , A.K. Jaiswal, S.K. Bhattacharya, Super oxide dismutase, catalase, glutathione peroxidase activity in brain of streptozotocin induced diabetic rat. Ind. J. Exp. Biol.(1999) 37 182-183.

14. M. Kinalski, A.Sledziewski, B. Jelejko, W. Zoerzycki, I. Kinalski, Acta Diabetol. (2000) 37 179-183.

15. Valko M, Rhodes CJ, Moncol J, Izakovic M, et al. Free radicals, metals and antioxidants in oxidative stress-induced cancer. Mini-review. Chem. Biol. Interact. (2006) 160 1-40.

16. J.Owoabi J, E.K.I Omogbai and O. Obasuyi, Antifungal and antibacterial activities of the ethanolic and aqueous extract of Kigella africana (Bignoniaceae) stem bark. Afr.J. Biotechnol. 6 (2007) 882-885.

17. R. Radha , M. Sermakkani and V. Thangapandian, Evaluation of Preliminary Phytochemical and Antimicrobial Activity of Andrographis echioides (L) Nees. International Journal of Pharmaceutical Science (2011) 2 S91-S101.

18. R. Murali, S. Srinivasan, N. Ashokkumar, Antihyperglycemic effect of fraxetin on hepatic key enzymes of carbohydrate metabolism in streptozotocin- induced diabetic rats, Bio chimie 95 (2013) 1848-1854.

19. P.Sibi, Ittiyavirah and Jahanara Hameed, Evaluation of anti-sress activity of Ethanolic extract of Alternanthera Sessilis (Linn.) and its Silver Nanoparticles In Chronic Variable Stress Model. World Journal Of Pharmacy And Pharmaceutical Sciences. (2012) 10 609-622.

20. R. Murali, S. Srinivasan, N. Ashokkumar, Antihyperglycemic effect of fraxetin on hepatic key enzymes of carbohydrate metabolism in streptozotocin- induced diabetic rats, Bio chimie 95 (2013) 1848-1854.

21. P.Trinder, Determination of glucose in blood using glucose oxidase with an alternative oxygen receptor, Ann. Clin. Biochem. (1969) 6, 24-27.

22. D.L Drabkin and J.H.Austin, Spectrophotomeric studies: Spectrophotometric constant for common hemoglobin derivatives in human, dogs and rabbit blood. Journal of Biological Chemistry (1932) 98 719.

23. S.Sudhakar Nayak and T.N. Pattabiraman, A new colorimetric method for the estimation of glycosylated haemoglobin. Clinica Chimica Acta. (1981) 109 267-274.

24. O.H.Lowry, N.J.Rosebrough, A.L.Farr and R.J.Randall, Protein measurement with the Folin phenol reagent. J Biol Chem. (1951) 193 265-275.

25. M.A. Morales, A.J. Jabbagy and H.P Tenenzi, Mutations affecting accumulation of glycogen. Neurospora News Lett. (1973) 20 24-25.

26. N. Brandstrup, J.E.Kirk and C.Bruni, The hexokinase and phosphoglucoisomerase activities of aortic and pulmonary artery tissue in individuals of various ages. J Gerontol. 12 (1957)166-171.

27. H. Koide, T. Oda, Pathological occurrence of glucose-6-phosphatase in liver disease. Clin Chim Acta. (1959) 4554-561.

28. J.M. Gancedo, C.Gancedo, Fructose 1, 6-bisphophatase, phosphofructokinase and glucose 6-phosphate dehydrogenase from fermenting yeast. Arch Microbiol. (1971) 76 132-138.

29. H.U.Bergmeyer, Glucose 6-phosphate dehydrogenase. Methods of enzymatic analysis. Verlag. Chemie. Weiheim., (1984) 2 222- 223.

30. J. Folch, I. Ascoli , M. Lees, J.A. Meath and F.N. LeBaron, Preparation of lipid extracts from brain tissue, J. Biol. Chem. (1951) 191: 833.

31. C.C Allain, Enzymatic determination of total serum cholesterol. Clin Chem. (1974) 20 470-475.

32. C. Izzo and F. Grillo, Murador E.Improved method for the determination of high density lipoprotein cholesterol I. Isolation of high- density lipoproteins by the use of polyethelene glycol 6000. Clin Chem. (1981) 27 371-374.

33. W.T. Friedewald, R.J Levy and D.S.Fredricken, Estimation of HDL-C in the plasma without the use of preparative ultracentrifuge. Clin Chem. (1972) 18449.

34. K.Falholt, W.Falholt and B. Lund, An easy colourimetric method for routine determination of free fatty acids in plasma. Clin. Chim. Acta. (1973) 46105.

35. M.W. McGowan, J.D. Artiss, D.R. Strandberg, and B.Zak, A peroxidase-coupled method for the colourimetric determination of serum triglycerides. Clin Chem. (1983) 29 538-542.

36. D.B. Zilversmit and A.K. Davis, Micro determination of phospholipids by TCA precipitation. J. Lab. 
Clin. Med. (1950) 35 155-161.

37. J.G.Reinhold, Mannual determination of serum total protein albumin and globulin fractions by Buiret method. In Standard Method of Clinical Chemistry (ed) M. REINER; Academic Press, New York, (1953) pp 88.

38. S. Reitman and S. Frankel, Glutamic - pyruvate transaminase assay by colorimetric method. Am. J. Clin. Path. (1957) 2856.

39. P.R.N. Kind and E.J King, Estimation of plasma phosphatase by determination of hydrolised phenol with amino-antipyrine. J.Clin. Pathol. (1954) 7 322-326.

40. S.B. Rosalki and D. Rau, Serum -glutamyl transpeptidase activity in alcoholism. Clin Chim Acta., (1972) 39 41-47.

41. J.K Fawcett and J.E. Scott, Determination of urea by urease method using Berthelot reaction. Journal of Clinical Pathology. (1960) 13, 156.

42. W.T. Caraway, Amer. J. clin. Path., 25, 840. 1963. In Standard Methods of Clinical Chemistry, Academic Press, New York edited by D. Seligson. (1955) 4 239-247.

43. N.W. Tietz, Fundamentals of Clinical Chemistry. Philadelphia, PA, WB Saunders. (1987) 638.

44. K. Yagi Methods For Estimating Lipid Peroxidation: An Analysis Of Merits And Demerits Indian Journal of Biochemistry \& Biophysics Chem Phys Lipids.(1987) 45 337-351

45. C.G. Fraga, B.E.Leibovitz \& A.L. Tappel,Free Radical Biol. Med.(1988) 4 155-161.

46. Z.Y. Jiang, J.V.Hunt and S.P. Wolff, Fundamentals of Clinical Chemistry Anal Biochem.(1992) 202 384.

47. P. Kakkar, B. Das, P.N. Viswanathan, A modified spectrophotometric assay of superoxide dismutase. Indian J Biochem Biophys. (1984) 21 130-2.

48. K.S. Sinha, Colometric assay of catalase. Annu.Rev. Biochem. (1972) 47 389-394.

49. J.J Rotruck and A.L.Pope, Selenium: biochemical role as a component of glutathione peroxidise. Science. (1973) 179 588-590.

50. G.L.Ellman, Tissue sulfhydryl groups. Arch. Biochem. Bio Phy.(1959) 82: 70-77.

51. S.T. Omaye, J.C.Turnabull, Sanberlick HE. Selected methods for the determination of ascorbic acid in animal cells, tissues and fluids. Methods Enzymol. (1979) 62 3-11.

52. H. Baker and O.Frankle, Plasma alpha tocopherol in man at various time intervals after ingesting free or acetylted tocopherol. Nutr. Rep.Int. (1980) 21 531-536.

53. Ali, S., Rohilla, A., Dahiya, A., Kushnoor, A. and Rohilla, S, Streptozotocin induced diabetes: mechanisms of induction. International Journal of Pharmaceutical Research and Development. (2011) $4011-015$.

54. Krishna, D., Rao, S. and Satyanarayana, M.L, Serum insulin levels and lipid profiles of streptozotocin induced diabetic wistar rats. Journal of Indian Veterinary Association, Keral. (2012) 10 22-26.

55. R.F. Doda, Diabetes mellitus, In: Kaplan LA, Amadeo JP, eds. Clinical Chemistry. St Louis: Mosby Year Book, (1996) 613-641.

56. Bamidele O, Arokoyo DS, Akinnuga AM, Oluwarole AO, Antidiabetic effect of aqueous extract of Basellaalba leaves and metformin in alloxan-induced diabetic albino rats. Afr. J. Biotechnol. (2014), 13, 2455-2458.

57. Kim, H.J., Lee, J.H., Kim, S.J., Oh, G.S., Moon, H.D., Kwon, K.B., Park, C., Park, B.H., Lee,H.K., Chung, S.Y, Roles of NADPH oxidases in cisplatin-induced reactiveoxygen species generation and ototoxicity. J. Neurosci. (2010), 30 3933-3946.

58. Olajire, A. A. and Azeez, L. Total antioxidant activity, phenolic, flavonoid and ascorbic acid contents of Nigerian vegetables. African Journal of Food Science and Technology. (2011) 2 022-029.

59. Nirmala A., Saraoja S., Gayathri Devi G. Antidiabetic activity of Basella rubra and its relationship with the antioxidant property. British biotechnology journal. (2011) 1 1-9.

60. Rathi S.S., Grover J.K. Vikrant V., and Biswas N.R. (2002). Prevention of experimental diabetic cataract by Indian Ayurvedic plant extract. Phytotherapy reserve. (2002) 16 774-777.

61. S. Sen, M. Kar, A. Roy, A.S. Chakraborti, Effect of nonenzymatic glycation on functional and structural properties of hemoglobin. Biophys Chem. (2005) 113 289-298.

62. V.Fonseca, Clinical significance of targeting postprandial and fasting hyperglycemia in managing type 2 diabetes mellitus.Curr Med Res Opin. (2003) 19 635-641.

63. G.E. Mortimore, C.E. Manton. Inhibition of insulin of valine turnover in liver. Journal of Biological Chemistry (1970) 245 2375-2383.

64. T.H. Dijk, V.H. van der Sluijs, C.H. Wiegman, J.F.W. Baller, L.A. Gustafson, H.J. Burger, A.W. Herling, F. Kuipers, A.J. Meijer and D.J. Reijngoud, Acute Inhibition of Hepatic Glucose-6- 
phosphatase Does Not Affect Gluconeogenesis but Directs Gluconeogenic Flux toward Glycogen in Fasted Rats. A pharmacological study with the chlorogenic acid derivative S4048. J. Biol. Chem. (2001) 276 25727-25735.

65. Z. Liu, E.J. Barrett, A.C. Dalkin, A.D. Zwart, J.Y. Chou, Effect of acute diabetes on hepatic glucose-6phosphatase activity and its messenger RNA level. Biochem. Biophys. Res. Commun. (1994) 205 680686.

66. P.N Pushparaj, B.K.Tan and C.H. Tan, The mechanism of hypoglycemic action of the semi-purified fractions of Averrhoa bilimbi in streptozotocin-diabetic rats. Life Sciences. (2001) 70 535-547.

67. M. Diaz-Flores, M.A. Ibanez-Hernandez, R.E. Galvan, M. Gutierrez, G. Duran-Reyes, R. MedinaNavarroo, D. Pascoe-Lira, C. Orttega-Camarillo, C. Vilar-Rojas, M. Cruz, L.A. Biaza-Gutman, Glucose-6- phosphste dehydrogenase activity and NADPH/NADP+ ratio in liver and pancreas are dependent on the severity of hyperglycemia in rat. Life Sci. (2006) 2601-2607.

68. K.N. Bopanna, J.Kannan, G.Sushma, R. Balaraman, S.P. Rathod, Antidiabetic and antihyperlipidemic effects of neem seed kernel powder on alloxan diabetic rabbits. Indian J. Pharmacol. (1997) 29 162-7.

69. Gordon T., Castelli W. P., Hjortland M. C., Kannel W. B., Dawber T. R. High density lipoprotein as a protective factor against coronary heart disease: The Framingham study. Am. J. Med. (1977) $62707-$ 714.

70. Mitra SK, Gopumadhavan S, Muralidhar TS, Anturlikar SD, Sujatha MB, Effect of D 400, a herbomineral preparation on lipid profile, glycatedhaemoglobin and glucose tolerance in streptozotocin induced diabetes in rats. Indian J. Exp. Biol. (1995) 33 798-800.

71. Oyedepo TA, Effect of Citrus maxima (Merr.) Fruit Juice in Alloxan-Induced Diabetic Wistar Rats. Sci. J. Med. Clin. Trials. (2012) Article ID sjmct-125, 8 Pages.

72. E. Rekka , P.Kourounakas, F. Shahidi, P.K. Janitha and P.D. Anasundara, Phenolic antioxidants. Critical Reviews in Food Science and Nutrition (1992) 32 67-103.

73. Azza OF., Afaf IA., and Mohamed GM. Toxicopathological effects of Guiera enegalensis extracts in wistar albino rats. J. Med. Plants Res. (2012) 3 699-702.

74. S.M. Rawi, A.Abdel-Moneim, O.M. Ahmed, Studies on the effect of garlic and glibenclamide on alloxan-diabetic rats. Egypt J Zool. (1998) 30 211-228.

75. D. Ramakrishna , G. Vidyasagar ,K. Pavan Kumar, I. Madhusudhana Reddy and S. Gupta Atyam , Evaluation of antidiabetic activity of Triumfetta Pilosa Roth In Streptozotocin induced Diabetic Rats. International Journal of Pharma Sciences and Research (2011) 2 98-103.

76. K. Rajaram , Antioxidant And Antidiabetic Activity of Tectona Grandis Linn. in Alloxan Induced Albino Rats. Asian J Pharm Clin Res. (2013) 6 174-177.

77. Y.P. Siu , K.T. Leung, M.K. Tong, T.H. Kwan, Use of allopurinol in slowing the progression of renal disease through its ability to lower serum uric acid level. Am J Kidney Dis. (2006) 47 51-59.

78. A.V. Krishnaraju,C.V. Rao, T.V.N. Rao, K.N. Reddy and Thrimuthu G, In vitro and in vivo antioxidant activity of Apharmamixis poly starchy bark. Am J Infect. Dis. (2009) 5 60-67.

79. M. Kinalski, A. ' Sledziewski, B. Telejko, W. Zarzycki, and I. Kinalska, "Lipid peroxidation and scavenging enzyme activity in streptozotocin-induced diabetes," Acta Diabetologica. (2000) 37179 183.

80. Pankaj Jain, Ashutosh Pareek, Yashumati Ratan, Swapnil Sharma, Sarvesh Paliwal Free Radicals and Dietary Antioxidants: A Potential Review Int. J. Pharm. Sci. Rev. Res.(2013) 18 34-48.

81. S. Lenzen , M. Tiedge, Flatt PR, Bailey CJ Panten U. Defective regulation of glucokinase in rat pancreatic islet celf tumors Acta Endocrinol (Copenh). (1979) 11 514-520.

82. P.R. Smith, P.J. Thornalley, Mechanism of the degradation of non-enzymatically glycated proteins under physiological conditions. Eur J Biochem. (1992) 210 729-39.

83. B. Halliwell and J.M.C. Gurteridge, The Antioxidants of human extracellular fluid,. Arch Biochem Biophys. (1990) 280 1-8.

84. S.M. Saumya and P.M. Basha, Antioxidant effect of Lager stroemia speciosapers (banaba) leaf extracting STZ induced diabetic mice. Indian journal of experimental biology (2011) 49 125-31.

85. B. Chance, H.Sies and A. Boveris, Hydroperoxide metabolism in mammalian organs. Physiological Reviews. (1979) 59, 527-605.

86. L. Flohé Glutathione peroxidase. Basic Life Sci. (1988) 49 663-668.

87. H.Imai, K. Narashima, M. Arai, H. Sakamoto, N. Chiba, Y. Nakagawa, 1998. Suppression of leukotriene formationin RBL-2H3 cells that overexpressed phospholipid hydroperoxide glutathione peroxidase. J. Biol. Chem. (1998) 273 1990-1997.

88. A. Meister Glutathione metabolism and its selective modification. J Biol Chem. (1988) 263 17205-8. 
89. P.A. Craven, F.R. DeRubertis, V.E. Kagan, R.K. Melhen and R.K. Studer. Effects of supplementation with vitamin c or E on albuminuria, glomerular TGF- $\beta$, and gloemarular size in Diabetes. Journal of the American society of Nephrology. (1997) 89 1465-1414.

90. P. Mukherjee, R. Bhattacharya, P. Wang, L.Wang, S. Basu, J.Nagy, A. Atala, D. Mukhopadhyay and S. Shay, Antiangiogenic properties of gold nanoparticles. Clin. Cancer Res. (2005) 11 3530-3534.

91. A. Samadder, A, S. Das J.Das, A. Paul, A and Khuda-Bukhsh, R, Ameliorative effects of Syzygium jambolanum extract and its poly (lactic-co-glycolic) acid nanoencapsulated form on arsenic-induced hyperglycaemic stress: a multi-parametric evaluation. J. Acupunc. Merid. Stud. (2012) 5310-318.

92. M. Afifi and A. M. Abdelazim, "Ameliorative effect of zinc oxide and silver nanoparticles on antioxidant system in the brain of diabetic rats," Asian Pacific Journal of Tropical Biomedicine. (2015) $10874-877$.

93. S. Sheweita, S. Mashaly, A. Newairy, H. Abdou, S.Eweda S . Changes in Oxidative Stress and Antioxidant Enzyme Activities in Streptozotocin-Induced Diabetes Mellitus in Rats: Role of Alhagi maurorumExtracts", Oxidative Medicine and Cellular Longevity. (2016) 1- 8. 